\title{
PRE-CONCEPTUAL DESIGN OF AUTOMATED SYSTEMS FOR SRF CAVITY ASSEMBLY AND OPTICAL INSPECTION*
}

\author{
T. Harden", M. Borden, A. Canabal, P. Pittman, T. Tajima \\ LANL, Los Alamos, NM 87544, U.S.A.
}

\begin{abstract}
The International Linear Collider (ILC) will require $\sim 20,000$ Superconducting Radio-Frequency (SRF) cavities. Improving the yield of high-gradient $(>35$ $\mathrm{MV} / \mathrm{m}$ ) cavities is currently one of the most critical issues for the ILC. LANL has been tasked to analyze SRF cavity failure and feedback the results to industry and academia. We have started a pre-conceptual design of an automated system for assembling and sealing the flanges on SRF cavities after high-pressure rinsing in a clean room. This should reduce particle contamination due to touch labor. We have also started a pre-conceptual design of an automated system to optically inspect precise locations (determined during testing) on the inner surface of SRF cavities for defects or contamination. This inspection will allow us to understand why a particular SRF cavity is substandard and potentially repair it. Using both automated systems should lead to field emission free cavities and higher yield of high-gradient cavities.
\end{abstract}

\section{INTRODUCTION}

While the SRF technology has been very successful [1], the largest number of the SRF cavities installed at a single site so far is only 338 for CEBAF at TJNAF in the early 1990s compared to the $\sim 20,000$ cavities required for the ILC. Moreover, the required accelerating gradient is $>30$ $\mathrm{MV} / \mathrm{m}$ compared to $5 \mathrm{MV} / \mathrm{m}$ for CEBAF. To build the ILC at a reasonable cost, the yield of successful cavities needs to be $>80 \%$ [2]. Unfortunately, the data from the DESY shows a large scatter in achieved gradients even in recent years, although the high end is increasing [3]. We believe that automated final rinsing and sealing of the cavities in a clean environment will help increase the reliability and reproducibility of the successful cavities once the prior chemical or electrolytic polishing is successful. Also, an automated inspection system will allow us to analyze defects that remain after polishing. This inspection will allow us to understand why a particular SRF cavity is substandard and potentially repair it.

\section{ASSEMBLY AUTOMATION}

The final assembly before a vertical test of SRF cavities consists of three major steps: to wash the cavity using ultra-pure water (an automatic process, see Figure 1 for LANL system), allow the cavity to air dry, and then attach sealing flanges to prevent contamination from entering the cavity. All of these operations take place in a Class 100 clean room.

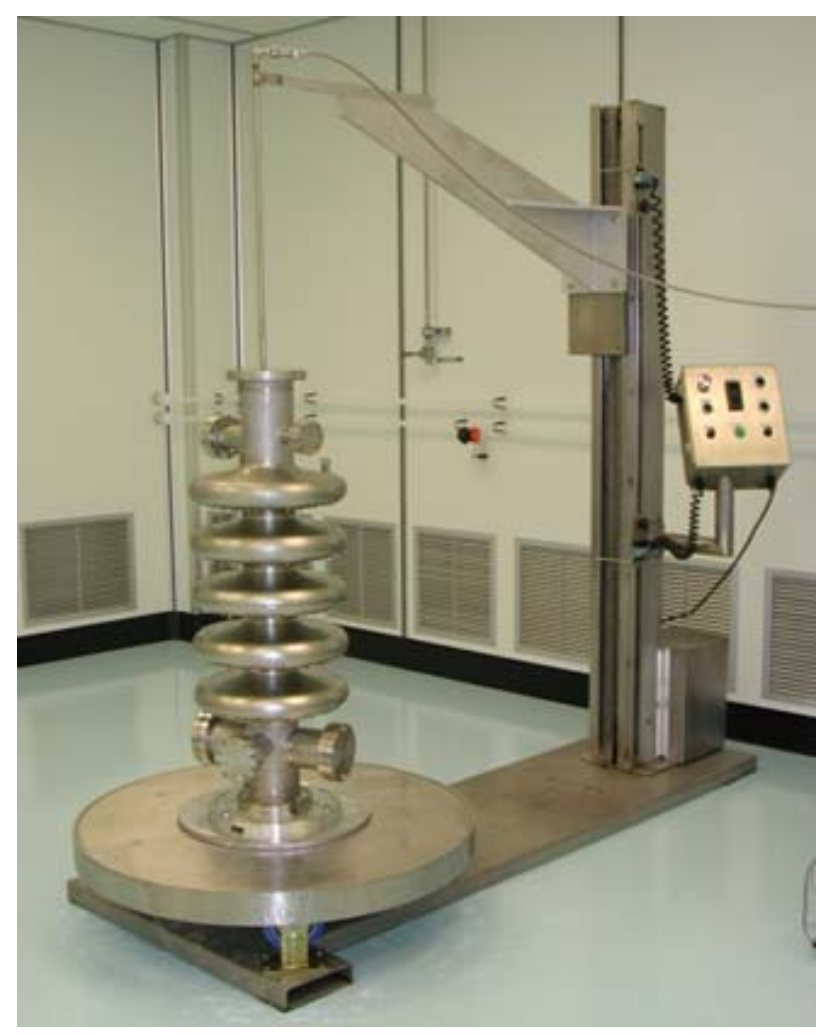

Figure 1: Automatic cavity rinsing station.

Even so, contamination is a major cause of substandard SRF cavities. Among other issues such as chemical residues and insufficient rinsing, it is believed that the manual installation of the sealing flanges is the primary source of this contamination. Therefore, we plan to replace the human with an automated system.

At the conceptual stage, we are considering two potential design configurations. The first configuration consists of a single robotic manipulator and clean fixture(s). The robot will position the clean, dry SRF cavity on the fixture(s) as required and then attach the sealing flange and gasket. The robot end-effector (EE) tooling will uniformly tighten the bolts holding each sealing flange in place to avoid vacuum leaks when the SRF cavity is immersed in $2 \mathrm{~K}$ superfluid helium. These steps will be repeated at each flange opening. Flanges will be attached from the bottom to minimize the chance of contamination entering the chamber. To reduce the amount of hardware required, a modified rinsing station may be used to provide some of the fixturing for this concept. Figures 2-4 illustrate this concept.

\footnotetext{
*Work supported by the US Department of Energy.

"harden@lanl.gov
} 


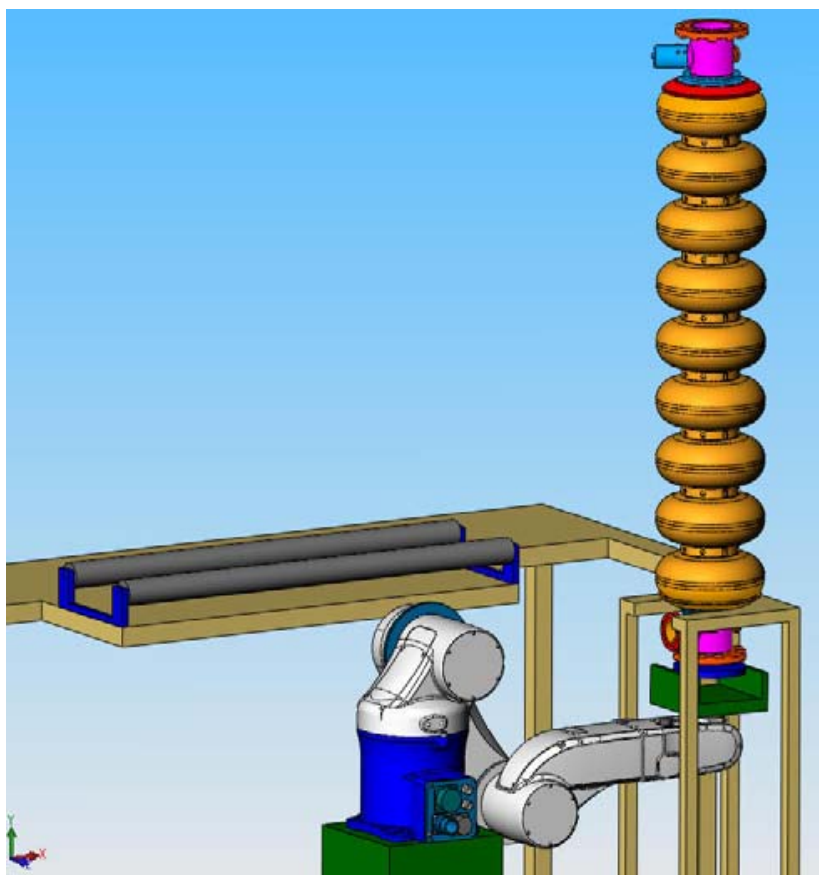

Figure 2: Attaching a flange to the end of the SRF cavity.

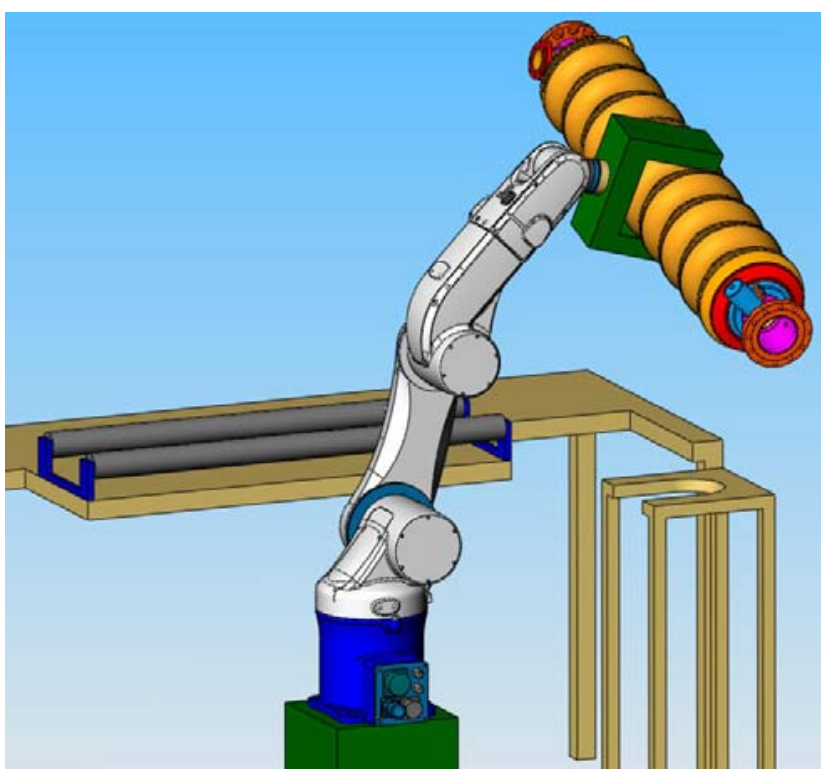

Figure 3: Positioning the SRF cavity for flange attachment.

The second potential configuration consists of two robotic manipulators. One manipulator will position the SRF cavity while the second manipulator performs the flange attachment operation. In this configuration, the second robot replaces most of the fixturing. This concept is also more flexible than the first concept since the SRF cavity can be positioned almost anywhere. Figure 5 illustrates this concept.

In either configuration, the robot(s) will be a commercial, off-the-shelf, Class 100 clean room rated manipulator. Any additional hardware (end-effectors, fixturing, etc.) will be clean room compatible or if unavailable, covered.

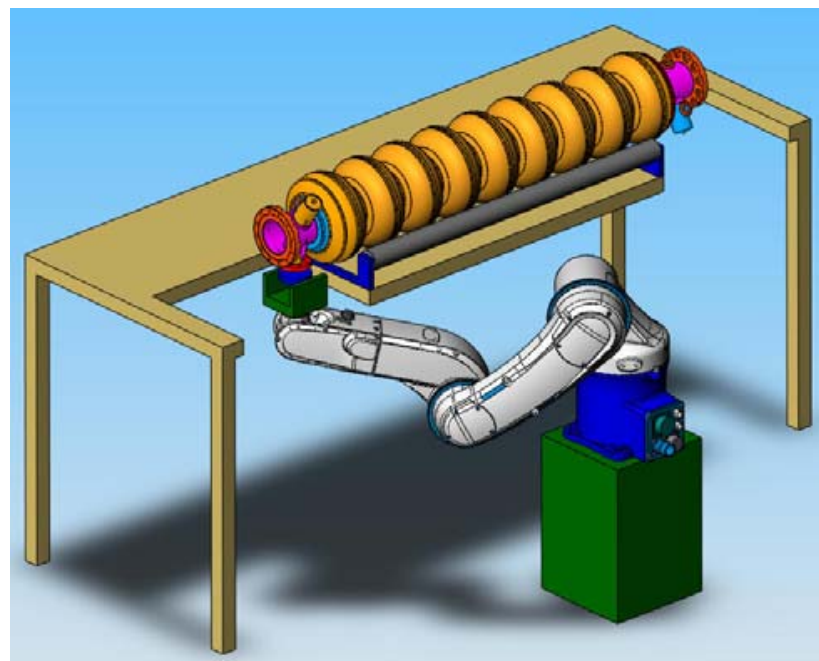

Figure 4: Attaching a flange to the side of the SRF cavity.

To ensure the cleanliness of assembly operations, particle counting sensors will be distributed within the workspace so that the assembly action is controlled based on the number of particles counted, e.g., when the number of particles exceeds the required number, the action stops until the particle count goes down to a specified number.

The system will be tied to a network so that it can send a notification to a pager, cell phone, and/or email address if something is not going well. Our ultimate goal is to run the system $24 / 7$ to increase production and reduce cost.

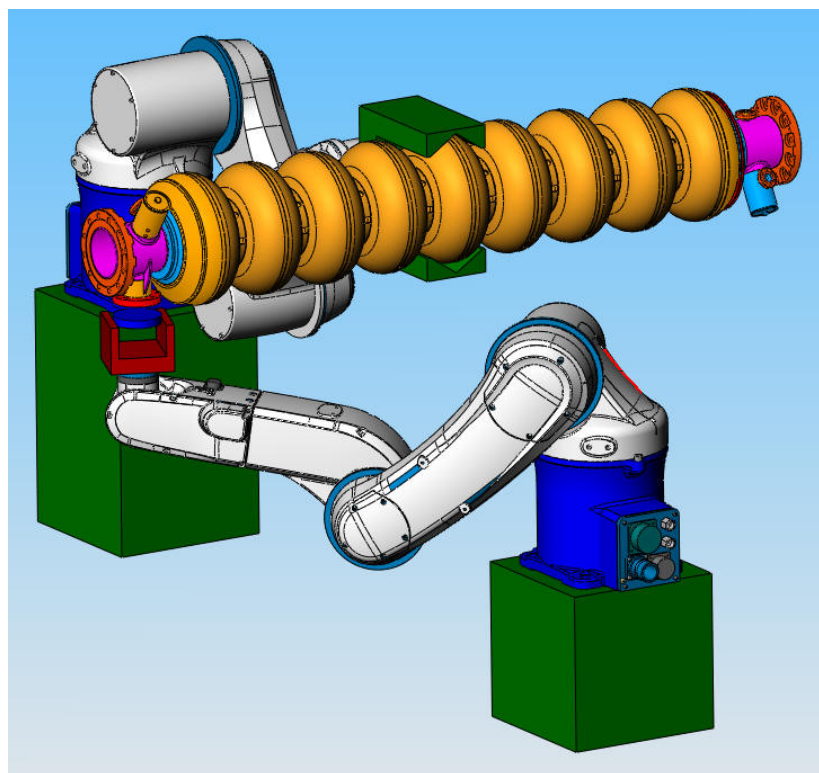

Figure 5: Two robot assembly concept.

\section{AUTOMATED INSPECTION SYSTEM}

Once assembly is complete, each SRF cavity must be tested to determine the field magnitude it can sustain. Substandard superconducting cavities quench at lower voltage / field strength. During testing, a temperature profile of the SRF cavity surfaces can be obtained. The warmest spot on this profile indicates the location where quenching started. If the cavity quenches at a low voltage/ 
field strength, this location is likely to have some sort of a defect (small scratch, contamination, weld defect, etc.).

The goal of automated inspection is to carefully inspect the precise location indicated by the temperature profile. This inspection will allow us to understand why the SRF cavity is substandard and potentially repair it.

Using a 10 micrometer resolution camera/scope, we plan to inspect the desired location. One potential configuration for the inspection system is to put an automated exoskeleton around a small commercial camera or video borescope. A concept view of this configuration is shown in Figure 6.

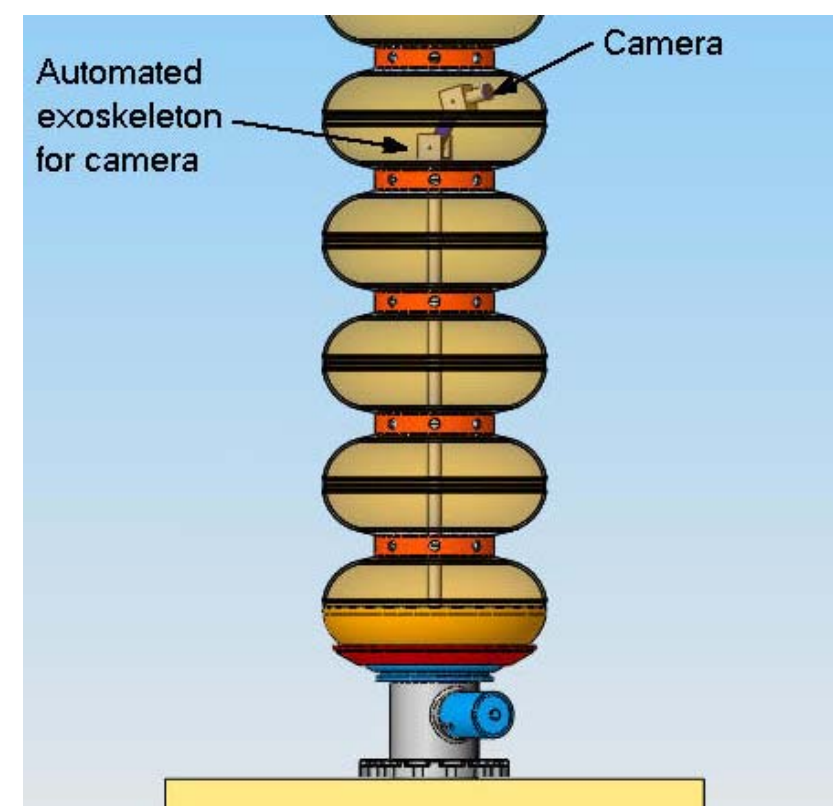

Figure 6: Inspection system concept.
In order to achieve the desired inspection resolution, special lighting optimized for inspecting a curved metal surface may be required. Also, manual adjustment of the focus and any zoom capability probably will be necessary.

Camera positioning will be achieved by developing/integrating either a custom or off-the-shelf software application that maps the quench point identified during SRF cavity testing to the required actuator motions to position the camera where desired.

Digital storage of the camera images will be integrated into the system. This will allow for easier and more complete analysis and characterization of potential defects after the inspection process is complete. It will also allow a defect database to be compiled. The defect database will provide a means to compare defects discovered in all of the SRF cavities that are inspected and potentially develop a science of defect types and repair techniques.

\section{REFERENCES}

[1] H. Padamsee, "Accelerating Applications of RF Superconductivity-Success Stories,” IEEE Trans. Appl. Superconductivity 15 (2005) 2432.

[2] The ILC Reference Design Report summary, http://www.linearcollider.org/pdf/RDR_Machine Overview_v5-1.pdf

[3] H. Padamsee, ILC Workshop, Bangalore, March 2007. 